\title{
Empirical photometric calibration of the Gaia red clump: Colours, effective temperature, and absolute magnitude ${ }^{\star}$
}

\author{
L. Ruiz-Dern, C. Babusiaux, F. Arenou, C. Turon, and R. Lallement
}

\begin{abstract}
GEPI, Observatoire de Paris, PSL Research University, CNRS UMR 8111, 5 place Jules Janssen, 92190 Meudon, France e-mail: laura.ruiz-dern@obspm.fr
\end{abstract}

Received 14 July 2017 / Accepted 16 October 2017

\begin{abstract}
Context. Gaia Data Release 1 allows the recalibration of standard candles such as the red clump stars. To use those stars, they first need to be accurately characterised. In particular, colours are needed to derive interstellar extinction. As no filter is available for the first Gaia data release and to avoid the atmosphere model mismatch, an empirical calibration is unavoidable.

Aims. The purpose of this work is to provide the first complete and robust photometric empirical calibration of the Gaia red clump stars of the solar neighbourhood through colour-colour, effective temperature-colour, and absolute magnitude-colour relations from the Gaia, Johnson, 2MASS, HIPPARCos, Tycho-2, APASS-SLOAN, and WISE photometric systems, and the APOGEE DR13 spectroscopic temperatures.

Methods. We used a 3D extinction map to select low reddening red giants. To calibrate the colour-colour and the effective temperature-colour relations, we developed a MCMC method that accounts for all variable uncertainties and selects the best model for each photometric relation. We estimated the red clump absolute magnitude through the mode of a kernel-based distribution function. Results. We provide 20 colour versus $G-K_{\mathrm{S}}$ relations and the first $T_{\text {eff }}$ versus $G-K_{\mathrm{S}}$ calibration. We obtained the red clump absolute magnitudes for 15 photometric bands with, in particular, $M_{K_{\mathrm{S}}}=(-1.606 \pm 0.009)$ and $M_{G}=(0.495 \pm 0.009)+(1.121 \pm$ 0.128) $\left(G-K_{\mathrm{S}}-2.1\right)$. We present a dereddened Gaia-TGAS HR diagram and use the calibrations to compare its red clump and its red giant branch bump with Padova isochrones.
\end{abstract}

Key words. stars: fundamental parameters - stars: abundances - stars: atmospheres - dust, extinction

\section{Introduction}

Measuring distances with high accuracy is as difficult as fundamental in astronomy. The most direct method for estimating astronomical distances is the trigonometric parallax. However, relative precisions of parallaxes decrease with distance. For distant structures we need to use standard candles such as red clump (hereafter RC) stars.

Red clump stars are low mass core $\mathrm{He}$-burning $(\mathrm{CHeB})$ stars that are cooler than the instability strip. These stars appear as an overdensity in the colour-magnitude diagram (CMD) of populations with ages older than $\sim 0.5-1 \mathrm{Gyr}$, covering the range of spectral types G8III - K2III with $4500 \mathrm{~K} \lesssim T_{\text {eff }} \lesssim 5300 \mathrm{~K}$. Indeed, the RC represents the young and metal-rich counterpart of the horizontal branch (see Girardi 2016, for a review).

The RC is used as a standard candle for estimating astronomical distances due to its relatively small dependency of the luminosity on the stellar composition, colour and age in the solar neighbourhood (Paczynski \& Stanek 1998; Stanek \& Garnavich 1998; Udalski 2000; Alves 2000; Groenewegen 2008; Valentini \& Munari 2010). As stated by Paczynski \& Stanek (1998), any method to obtain distances to large-scale structures suffers mainly from four problems: the accuracy of the absolute magnitude determination of the stars used, interstellar extinction, distribution of the inner properties of these stars (mass, age and chemical composition), and the size

* Full Table A.1 is only available at the CDS via anonymous ftp to cdsarc.u-strasbg. fr (130.79.128.5) or via

http://cdsarc.u-strasbg.fr/viz-bin/qcat?J/A+A/609/A116 of the sample. Whether the use of the RC may be considered particularly different than other standard candles such as RR Lyrae or Cepheids, is precisely due to their large number. The larger the sample used, the lower the statistical error in distance calculations. To efficiently use the RC as a standard candle, a good characterisation of the calibrating samples, here the solar neighbourhood, is needed, to which stellar population corrections can then be applied (see e.g. Girardi et al. 1998).

The first Gaia Data Release (GDR1) was delivered to the scientific community in September 2016 (Gaia Collaboration 2016). Although we will have new and more accurate astrometric and photometric measurements for thousands of RC stars in future releases, this first catalogue includes the Tycho-Gaia Astrometric Solution (TGAS) subsample (Lindegren et al. 2016) that already has a significant set of accurate solar neighbourhood $\mathrm{RC}$ parallaxes; the systematic error is at the level of 0.3 mas, i.e. three times better than in the HIPPARCos catalogue for 20 times more stars.

By comparing the observations with isochrones we can directly constrain stellar parameters such as ages and metallicities. However, we found that at the level of red giant stars, atmosphere models and observations do not fit: there is a gap between the models and observations no matter the photometric bands nor the atmosphere models used. As an example, we show this issue in Fig. 1 for the $B-V$ versus $V-I$ and $J-K_{\mathrm{s}}$ versus $V-K_{\mathrm{s}}$ colour-colour diagrams of some RC stars, and for both Padova (Parsec 2.7) and Dartmouth isochrones, which use ATLAS and Phoenix atmosphere models, respectively. A more exhaustive work on the important effects on the 

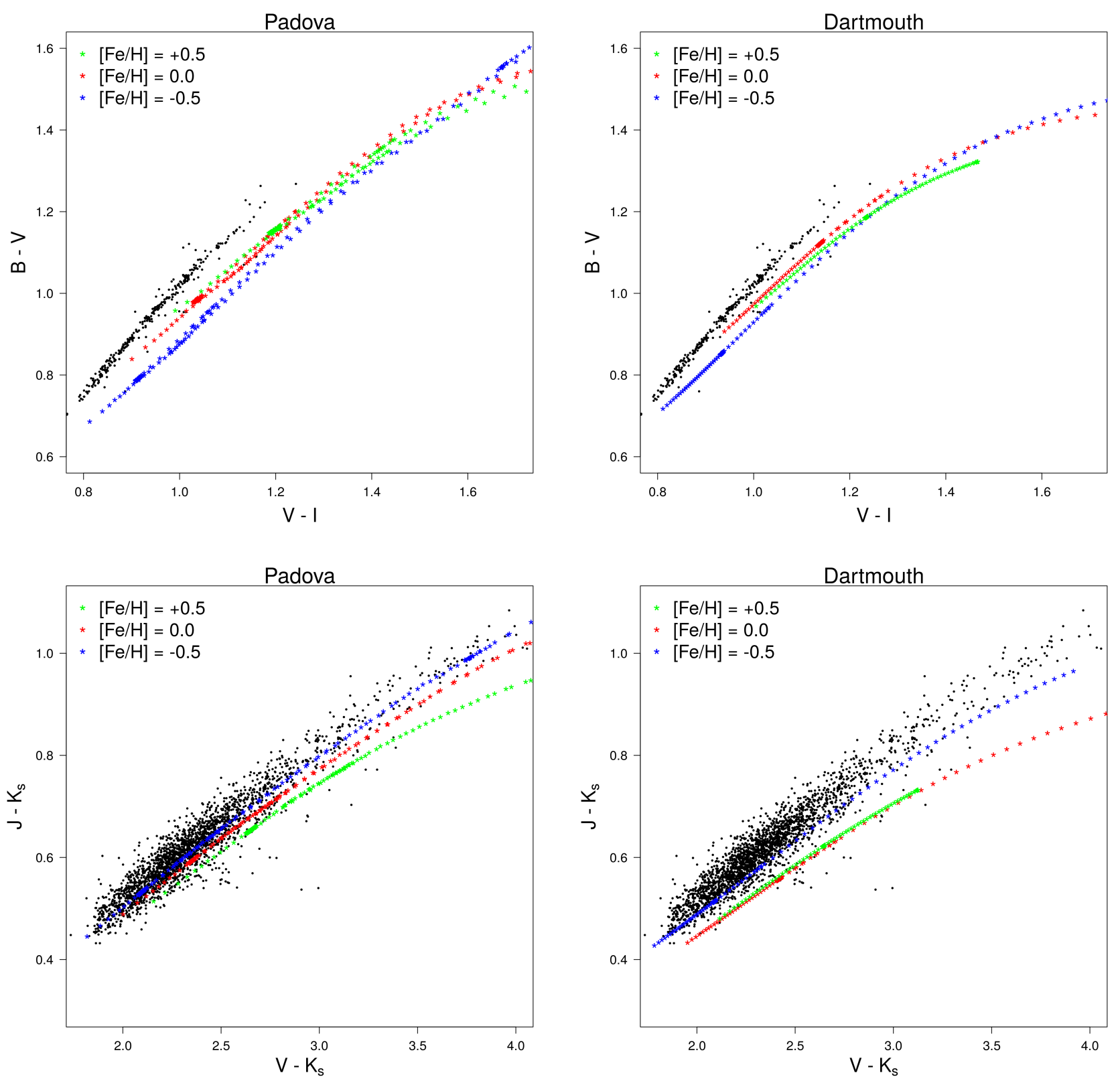

Fig. 1. $B-V$ vs. $V-I$ and $J-K_{\mathrm{S}}$ vs. $V-K_{\mathrm{S}}$ colour-colour diagrams of RC stars (sample described in Sect. 2). The median metallicity of the sample is about -0.2. Padova Parsec 2.7 (left) and Dartmouth (right) isochrones with a median age of 2 Gyr are overplotted for three different metallicities: (green) $[\mathrm{Fe} / \mathrm{H}]=+0.5$, (red) $[\mathrm{Fe} / \mathrm{H}]=0.0$, and (blue) $[\mathrm{Fe} / \mathrm{H}]=-0.5$. Only the red giant branch and the early assymptotic giant branch are shown.

choice of atmosphere models and other parameters may be found in Aringer et al. (2016). We checked that a unique shift is not enough to correct this gap because the slope is also different. We also checked the influence of filter modelling. Nevertheless, it seems that it is most probably an issue of atmosphere models.

Therefore there are two aspects that led us to develop the purely empirical calibrations that we present in this work: first, the need for photometric calibrations totally independent of models; and second, the fact that there is no on-board Gaia calibrated filter profile (instrumental response) available for the GDR1, thus a colour-colour calibration was automatically needed. Jordi et al. (2010) already predicted some colour relationships based on theoretical spectra and the nominal Gaia passbands (calibrated before launch), but the effective filters actually differ slightly (van Leeuwen et al. 2017). Therefore, there is a special interest in using colour-colour empirical calibrations instead.

In this work we present the first metallicity-dependent empirical colour-colour (hereafter $C C$ ), effective temperature ( $T_{\text {eff-colour and colour- }} T_{\text {eff }}$, hereafter $T_{\text {eff }} C$ and $C T_{\text {eff }}$, respectively) and absolute magnitude $\left(M_{G}\right.$ and $\left.M_{K}\right)$ calibrations for solar neighbourhood RC stars using the Gaia $G$ magnitude.

The paper is organised as follows. In Sect. 2 we describe the sample selection, the adopted constraints and how the interstellar extinction has been handled. The method developed to calibrate all the $C C$ and $T_{\text {eff }}$ relations is explained in Sect. 3. The calibrations obtained are presented in Sect. 4. In Sect. 5 we detail the RC absolute magnitude calibration. And finally in Sect. 6 we present the dereddened TGAS HR diagram and compare its RC to the Padova isochrones. 


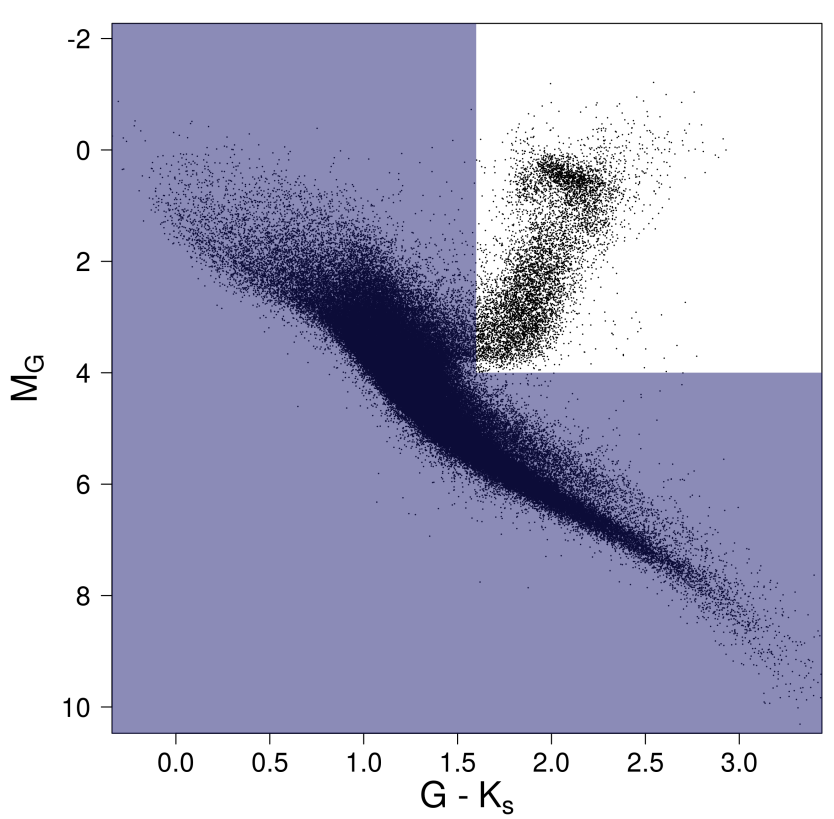

Fig. 2. TGAS HR diagram with parallax precision $\leq 10 \%, \sigma_{G}<0.01$, $E_{B-V}<0.015$, and 2 MASS $J K_{\mathrm{s}}$-bands high photometric quality (data available in Table A.1, Appendix A). The non-shaded region corresponds to the selection of red giants with $G-K_{\mathrm{S}}>1.6$ and $M_{G}<4.0$.

\section{Sample selection}

Different samples were constructed using TGAS data for the colour-colour and the effective temperature calibrations. To ensure their quality we considered the following constraints.

\subsection{Interstellar extinction}

One of the main issues with $C C, T_{\text {eff }} C$ and $C T_{\text {eff }}$ calibrations for giants is the extinction handling. To select low extinction stars, we use here the most up-to-date 3D local extinction map of Lallement et al. (2014), Capitanio et al. (2017), together with the 2D Schlegel et al. (1998) map for stars for which the distance goes beyond the 3D map borders. We scaled the Schlegel et al. (1998) map values by 0.884 according to Schlafly \& Finkbeiner (2011) and in agreement with the Capitanio et al. (2017) $E(B-$ $V)$ scale. We fixed a maximum threshold of 0.01 in $E(B-V)$, i.e. 0.03 in $A_{0}$, for a maximum distance corresponding to a parallax $\varpi-\sigma_{\varpi}$. Such a selection of low extinction stars should lead to more robust results than a dereddening that would be dependent not only on an extinction map but also on an extinction law and could lead to either over or under correction of the extinction.

\subsection{Selection of red giants}

To select solar neighbourhood red giant stars we considered the following two criteria:

$G-K_{\mathrm{s}}>1.6$

$m_{G}+5+5 \log _{10}\left(\frac{\varpi+2.32 \sigma_{\varpi}}{1000}\right)<4.0$.

The factor 2.32 on the parallax error corresponds to the 99th percentile of the parallax probability density function. Figure 2 shows the selected region on the HR diagram. The data used to construct this HR diagram is described in Table A.1. see Sect. 6 for more details on the $\mathrm{RC}$ region of this diagram.
We extended the parallax criteria to cover the full red giant branch so that our calibrations have a larger interval of applicability than just the RC. We checked that this large magnitude interval did not have any significant impact on the calibration. The fit is, on the contrary, very sensitive to red dwarf stars contaminants. Indeed the slope of giants and dwarfs in colour-colour distributions changes gradually as the stars are cooler (e.g Bessell \& Brett 1988). A selection based on spectroscopic surface gravity $(2.5<\log g<3.5)$ was tested and discarded because of the non-negligible percentage of giants/dwarfs misidentification in some surveys; for example, we found $\sim 2 \%$ misidentified RAVE stars when selecting those matching Appendix A criteria that are supposed to be inside the non-shaded region of Fig. 2. The chosen parallax criteria allows us to guarantee there is no contamination of dwarfs in our sample.

\subsection{Photometric data}

Our calibrations aim to cover all major visual and infrared bands. To achieve this we selected only those DR1 stars that have photometric information (with uncertainties) from the following catalogues: GDR1, HIPPARCOS, Tycho-2, 2MASS, APASS DR9, and WISE.

\subsubsection{GDR1}

We included stars with uncertainties lower than 0.01 mag in the $G$ band. An error of $10 \mathrm{mmag}$ was quadratically added to mitigate the impact of bright stars residual systematics; see Arenou et al. (2017), Evans et al. (2017).

\subsubsection{HIPPARCOS}

We selected stars with $B, V$, and $H_{\mathrm{p}}$ bands with uncertainties lower than 0.03 mag. We did not include the $I$ band because of the low number $(\sim 12)$ of remaining stars when selecting those with $V-I$ direct measurements in the Cousins system (field $H 42=A$ ), with measurements in the Johnson system then converted to the Cousins system (field $H 42=C$ ), and with measurements in the Kron-Eggen system then converted to the Cousins system (field $H 42=E$ ). For more details see Perryman et al. (1997), Vol. 1, Sect. 1.3, Appendix 5.

\subsubsection{Tycho-2}

We selected stars with $B_{\mathrm{T}}$ and $V_{\mathrm{T}}$ photometric bands (Høg et al. 2000) with uncertainties lower than 0.03 mag.

\subsubsection{MASS}

We included those stars with $J, H$, and $K_{\mathrm{s}}$ photometry (Cutri et al. 2003) from the cross-matched 2MASS-GDR1 catalogue (Marrese et al. 2017) with high photometric quality (i.e. flag q2M = A) and from Laney et al. (2012). Only stars with uncertainties lower than 0.03 mag were chosen.

\subsubsection{APASS DR9}

We also considered stars with $g, r$, and $i$ bands (Henden et al. 2016) cross-matched with Gaia at 2 " precision, and only stars with standard deviations obtained from more than one observation $\left(u_{e}=0\right.$ flag in the APASS catalogue) and uncertainties lower than 0.03 mag. Duplicates were removed by keeping the 
source with the largest number of photometric bands provided in APASS.

\subsubsection{WISE}

We selected stars with $W 1, W 2, W 3$, and $W 4$ photometry (Wright et al. 2010) from the cross-matched WISE-GDR1 catalogue (Marrese et al. 2017) with uncertainties lower than $0.05 \mathrm{mag}$, high photometric quality (i.e. flag qph $=\mathrm{A}$ ), low probability of being true variables (i.e. flag var $<7$ ), a source shape consistent with a point source (i.e. flag ex $=0$ ) and showing no contamination from artefacts (i.e. flag ccf $=0$ ). According to Cotten \& Song (2016), for $W 2$ we also removed stars brighter than $7 \mathrm{mag}$, because they are saturated.

\subsection{Binarity and multiplicity}

We removed all stars flagged as binaries and belonging to multiple systems. To do so we took into account the specific flags in the HIPPARCOS catalogue and the last updated information from the 9th Catalogue of Spectroscopic Binary Orbits (SB9, Pourbaix et al. 2004, 2009), the Tycho Double Star Catalogue (TDSC, Fabricius et al. 2002), and Simbad database (stars with flag “**”). We also considered only stars for which the proper motions from HIPPARCos are consistent with those of Tycho2 (rejection $p$-value: 0.001 ). According to a specific test carried out in the framework of the Gaia data validation team (Arenou et al. 2017) most of the stars for which the proper motions are not consistent between both catalogues are expected to be long period binaries not detected in HIPPARCOS, and for which the longer time baseline of Tycho- 2 could have provided a more accurate value.

\subsection{Metallicity}

We selected stars with metallicity information from various sources, since there were not enough stars when using just one reference. The expected effect of heterogeneity is that the differences between all the measurements will increase the dispersion of the residuals and decrease the dependence of the calibrations with metallicity. Noting in brackets the percentage of stars found and used in this work ${ }^{1}$, our established priority order is: Morel et al. (2014) [0\%], Thygesen et al. (2012) [0\%], Bruntt et al. (2012) [0.04\%], Maldonado \& Villaver (2016) [0.6\%], Alves et al. (2015) [1.4\%], Jofré et al. (2015) [0.4\%], Bensby et al. (2014) [0.4\%], da Silva et al. (2015) [0.04\%], Mortier et al. (2013) [0.04\%], Adibekyan et al. (2012) [0.3\%], APOGEE DR13 (SDSS Collaboration 2017) [9.9\%], GALAH (Martell et al. 2017) [0.4\%], Ramírez et al. (2014a) [0\%], Ramírez et al. (2014b) [0\%], Ramirez et al. (2013) [0.04\%], Zieliński et al. (2012) [0.2\%], Puzeras et al. (2010) [0.04\%], Takeda et al. (2008) [0.08\%], Valentini \& Munari (2010) [0.6\%], Saguner et al. (2011) [0\%], RAVE DR5 (Kunder et al. 2017) [67.6\%], LAMOST DR2 (Luo et al. 2016) [13.5\%], AMBRE DR1 (De Pascale et al. 2014) [1.0\%], Luck (2015) [0.7\%], and PASTEL (Soubiran et al. 2016) [2.8\%].

The final sample contained 2334 stars when considering the extinction, red giants selection, multiplicity, metallicity and the photometric constraints on the $G$ and $K_{\mathrm{s}}$ bands. Subsamples were then generated for each colour-colour relation depending on the other photometric bands used (see later in Table 4 the final sizes for every fit).

\footnotetext{
1 Some references used in our compilation could lead to no star in the final sample, i.e. $0 \%$ owing to the various quality cuts
}

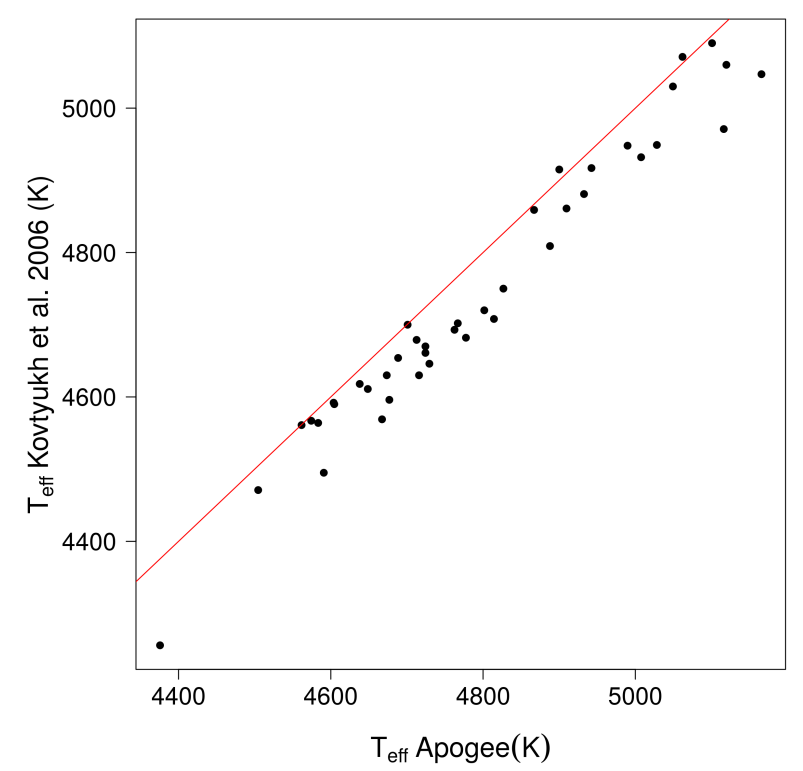

Fig. 3. Comparison of the spectroscopic effective temperatures of the 41 stars in common between APOGEE and Kovtyukh et al. (2006).

\subsection{Effective temperature}

For the $T_{\text {eff }}$ calibrations the largest homogeneous sample filling all the above criteria is the 13th release (DR13) of the APOGEE survey (Holtzman et al. 2015; García Pérez et al. 2016; SDSS Collaboration 2017). To increase the sample size, we also included stars not in TGAS with APOGEE $\log g<3.2$, using therefore only the Schlegel et al. (1998) map to apply our low extinction criteria. The weighted mean of the parameters was computed for the duplicated sources. The cross-match with Gaia was carried out through the 2MASS cross-match (Marrese et al. 2017) with an angular distance $<1$ ". The final sample contained 530 stars.

The SDSS Collaboration discusses a systematic offset ${ }^{2}$ of their spectroscopic effective temperatures from photometrically derived temperatures for metal-poor stars (by as much as 200$300 \mathrm{~K}$ for stars at $[\mathrm{Fe} / \mathrm{H}] \sim-2)$. Consequently the authors provided a correction as a function of metallicity. We decided not to apply their suggested correction as it is based on comparison with photometric temperatures. We compared the APOGEE temperatures to the PASTEL temperatures and found metallicity correlations only for the most metal-poor stars $([\mathrm{Fe} / \mathrm{H}]<-1.5)$. We tested that our calibrations did not change significantly when we removed the most metal-poor stars from our sample.

For metal-rich stars, we compared 41 giant stars (within $-0.4<[\mathrm{Fe} / \mathrm{H}]<0.2)$ from Kovtyukh et al. (2006) in common with APOGEE. These authors got a very good internal precision of 5-20 K (zero-point difference expected to be smaller than $50 \mathrm{~K})$. As shown in Fig. 3 we find a difference of about $50 \mathrm{~K}$ with respect to APOGEE with no correlation with $[\mathrm{Fe} / \mathrm{H}]$ and a dispersion in agreement with the precisions provided in both catalogues.

\section{Calibration method}

To derive accurate photometric relations, we implemented a Monte Carlo Markov Chain (MCMC) method, which allows us

\footnotetext{
2 http://wWw.sdss.org/dr13/irspec/parameters/ \#QualityoftheASPCAPStellarParameters
} 
to account and deal with the uncertainties of both the predictor and response variables in a robust way.

We provide all the calibrations with respect to the $G-K_{\text {s }}$ colour. Those photometric bands will be widely used thanks to the all-sky and high uniformity properties of the Gaia and 2MASS catalogues. Thus, in this work we provide the following calibrations:

Colour $=f\left(G-K_{\mathrm{s}},[\mathrm{Fe} / \mathrm{H}]\right)$

$\hat{T}=f\left(G-K_{\mathrm{s}},[\mathrm{Fe} / \mathrm{H}]\right)$

$G-K_{\mathrm{s}}=f(\hat{T},[\mathrm{Fe} / \mathrm{H}])$

where Colour includes all possible combinations of the photometric bands considered in this work (Sect. 2.3), and $\hat{T}=$ $T_{\text {eff }} / 5040$ is the normalised effective temperature.

\subsection{Polynomial models}

The general fitting formula adopted is

$Y=a_{0}+a_{1} X+a_{2} X^{2}+a_{3}[\mathrm{Fe} / \mathrm{H}]+a_{4}[\mathrm{Fe} / \mathrm{H}]^{2}+a_{5} X[\mathrm{Fe} / \mathrm{H}]$

which is a second order polynomial ${ }^{3}$ where, following Eq. (3), $X$ is either the $G-K_{\mathrm{s}}$ or the normalised effective temperature $\hat{T}$, $Y$ is (for $C C$ ) a given colour to be calibrated or (for $T_{\text {eff }}$ relations) either $G-K_{\mathrm{s}}$ or $\hat{T}$, and $a_{i}$ are the coefficients to be estimated. In order to provide the most accurate fit for each relation, the process (see Sect. 3.3) penalises by the complex terms so that, in the end, seven different models may be tested for every relation (Model 7 being the more complex model) as follows:

Model 1: $Y=a_{0}+a_{1} X$

Model 2: $Y=a_{0}+a_{1} X+a_{2} X^{2}$

Model 3: $Y=a_{0}+a_{1} X+a_{3}[\mathrm{Fe} / \mathrm{H}]$

Model 4: $Y=a_{0}+a_{1} X+a_{2} X^{2}+a_{3}[\mathrm{Fe} / \mathrm{H}]$

Model 5: $Y=a_{0}+a_{1} X+a_{3}[\mathrm{Fe} / \mathrm{H}]+a_{4}[\mathrm{Fe} / \mathrm{H}]^{2}$

Model 6: $Y=a_{0}+a_{1} X+a_{2} X^{2}+a_{3}[\mathrm{Fe} / \mathrm{H}]+a_{4}[\mathrm{Fe} / \mathrm{H}]^{2}$

Model 7: $Y=a_{0}+a_{1} X+a_{2} X^{2}+a_{3}[\mathrm{Fe} / \mathrm{H}]+a_{4}[\mathrm{Fe} / \mathrm{H}]^{2}$

$$
+a_{5} X[\mathrm{Fe} / \mathrm{H}] \text {. }
$$

Input uncertainties from all variables are taken into account in the model.

\subsection{Monte Carlo Markov Chain}

A MCMC was run for every model tested with 10 chains and 10000 iterations for each. We used the runjags ${ }^{4}$ library from the $\mathrm{R}$ program language. An uninformative prior was set through a normal distribution with zero mean and standard deviation 10 . Further, we also set an initial value for every coefficient. That is, we used the output coefficients obtained for each model through a multiple linear regression; this is a simpler method that does not take uncertainties into account, but allows to obtain approximated values. The MCMC fit is run on the standardised variables to improve the efficiency of MCMC sampling (reducing the autocorrelation in the chains). Chain convergence is checked with the Gelman and Rubin convergence diagnostic.

\footnotetext{
3 Upper degrees were tested, but discarded by an analysis of variance test (ANOVA), meaning that simpler models were good enough to describe the data

4 https://cran.r-project.org/web/packages/runjags/ runjags.pdf
}

\subsection{Best model selection: deviance information criterion}

The model selection was carried out through a process of penalisation by the complex terms. To do so we took advantage of the deviance information criterion (DIC) (Plummer 2008), and we tested the models in pairs. A given complex model was compared to the next simpler model; for example, we removed the highest order interactions, starting with the cross-term $X *[\mathrm{Fe} / \mathrm{H}]$.

The method continuously determined the next pair of models to be compared, ran the MCMC for each and checked their DIC. When the DIC difference was significantly negative at $1 \sigma$ (i.e. $\left.\Delta \mathrm{DIC}+\sigma_{\Delta \mathrm{DIC}}<0\right)$ the complex model was kept, or else the next pair was tested.

\subsection{Outliers}

Once the best model was determined, the method checked whether there were calibrated stars $3 \sigma$ away from the model. If so, the furthest star was removed and the complete process was run again. Outliers were eliminated one by one to ensure that the further outlier was not causing a deviation in the model that led us to consider other stars as "false outliers".

\section{Calibration results}

\subsection{Colour-colour relations}

Table 1 gives the coefficients for each of the 20 colour versus $G-K_{\mathrm{s}}$ fit, together with the $G-K_{\mathrm{s}}$ and metallicity ranges of applicability, which are defined by the maximum and minimum values of each individual sample, along with the number $(N)$ of stars used after the $3 \sigma$ clipping, the percentage of outliers removed, and the final root mean square deviation (RMS). Figure 4 shows the colour versus $G-K_{\mathrm{s}}$ relations obtained for 4 of the 20 colour indices with the residuals of the fit as a function of the colour itself and the metallicity. The scatter obtained in the residuals is very small ( $\sim \pm 0.03$ globally).

\subsection{Effective temperature calibration}

Table 2 provides the coefficients of the fit for the $\hat{T}$ versus $G-K_{\mathrm{s}}$ and the $G-K_{\text {s }}$ versus $\hat{T}$ calibrations. The colour, $T_{\text {eff }}$ and metallicity ranges of applicability are specified as well as the number $(N)$ of stars used after the $3 \sigma$ clipping, the percentage of outliers removed, and the final root mean square deviation.

Figure 5 shows the $\hat{T}$ versus $G-K_{\text {s }}$ relation obtained with the residuals as a function of $G-K_{\mathrm{s}}$ colour index and metallicity. Since previous works in the literature use $\theta=5040 / T_{\mathrm{eff}}$ instead of the $\hat{T}$ considered here (e.g. Ramírez \& Meléndez (2005), González Hernández \& Bonifacio (2009) or Huang et al. (2015)), we also computed the calibration by using $\theta$. We found both calibrations look similar except for the cool stars, for which we just have a few points. We may see how in this region the $\hat{T}$ relations at various metallicities cross each other in an unrealistic way. This does not happen for the $\theta$ fit. However, after having statistically compared both the $\hat{T}$ and $\theta$ calibrations, we chose to provide only the coefficients for $\hat{T}$ versus $G-K_{\mathrm{s}}$. Indeed, DIC is significantly lower for the $\hat{T}$ fit. The dispersion obtained on the $T_{\text {eff }}$ residuals is about $59 \mathrm{~K}$, which is consistent with the uncertainties of the APOGEE data used ( $\sim 69 \mathrm{~K})$. 
Table 1. Coefficients and range of applicability of colour versus $G-K_{\mathrm{S}}$ relations, $Y=a_{0}+a_{1}\left(G-K_{\mathrm{S}}\right)+a_{2}\left(G-K_{\mathrm{S}}\right)^{2}+a_{3}[\mathrm{Fe} / \mathrm{H}]+a_{4}[\mathrm{Fe} / \mathrm{H}]^{2}+$ $a_{5}\left(G-K_{\mathrm{S}}\right)[\mathrm{Fe} / \mathrm{H}]$.

\begin{tabular}{|c|c|c|c|c|c|c|c|c|c|c|c|}
\hline Colour & $G-K_{\mathrm{s}}$ range & {$[\mathrm{Fe} / \mathrm{H}]$ range } & $a_{0}$ & $a_{1}$ & $a_{2}$ & $a_{3}$ & $a_{4}$ & $a_{5}$ & RMS & $\%_{\text {outliers }}$ & $N$ \\
\hline$B-G$ & {$[1.6,2.4]$} & {$[-1.4,0.4]$} & $0.583 \pm 0.180$ & $-0.046 \pm 0.187$ & $0.215 \pm 0.049$ & $0.144 \pm 0.006$ & - & - & 0.02 & 17.9 & 230 \\
\hline$B-V$ & {$[1.6,2.4]$} & {$[-1.4,0.4]$} & $-0.094 \pm 0.017$ & $0.552 \pm 0.009$ & - & $0.129 \pm 0.005$ & - & - & 0.02 & 10.4 & 251 \\
\hline$B-J$ & {$[1.6,2.4]$} & {$[-1.5,0.4]$} & $-0.117 \pm 0.041$ & $1.432 \pm 0.021$ & - & $0.153 \pm 0.011$ & - & - & 0.03 & 12.9 & 176 \\
\hline$B-K_{\mathrm{s}}$ & {$[1.6,2.4]$} & {$[-1.5,0.4]$} & $-0.161 \pm 0.038$ & $1.757 \pm 0.020$ & - & $0.141 \pm 0.011$ & - & - & 0.02 & 9.3 & 254 \\
\hline$G-H_{\mathrm{p}}$ & {$[1.6,2.4]$} & {$[-1.5,0.4]$} & $0.029 \pm 0.009$ & $-0.270 \pm 0.005$ & - & $-0.023 \pm 0.003$ & - & - & 0.01 & 5.3 & 270 \\
\hline$G-V$ & {$[1.6,2.4]$} & {$[-1.5,0.4]$} & $-0.286 \pm 0.104$ & $0.191 \pm 0.107$ & $-0.110 \pm 0.028$ & $-0.017 \pm 0.003$ & - & - & 0.01 & 3.9 & 274 \\
\hline$G-B_{\mathrm{T}}$ & {$[1.6,2.4]$} & {$[-1.4,0.4]$} & $-0.375 \pm 0.257$ & $-0.194 \pm 0.267$ & $-0.218 \pm 0.069$ & $-0.201 \pm 0.009$ & - & - & 0.03 & 12.7 & 241 \\
\hline$G-V_{\mathrm{T}}$ & {$[1.6,2.4]$} & {$[-1.5,0.4]$} & $-0.261 \pm 0.115$ & $0.122 \pm 0.119$ & $-0.109 \pm 0.031$ & $-0.034 \pm 0.006$ & $-0.016 \pm 0.007$ & - & 0.01 & 3.5 & 272 \\
\hline$G-J$ & {$[1.6,3.6]$} & {$[-4.8,1.0]$} & $0.256 \pm 0.021$ & $0.510 \pm 0.019$ & $0.027 \pm 0.004$ & $0.016 \pm 0.002$ & $0.005 \pm 0.001$ & - & 0.02 & 0.2 & 2178 \\
\hline$V-J$ & {$[1.6,2.4]$} & {$[-1.5,0.4]$} & $-0.028 \pm 0.026$ & $0.880 \pm 0.013$ & - & - & - & - & 0.03 & 2.4 & 200 \\
\hline$V-K_{\mathrm{s}}$ & {$[1.6,2.4]$} & {$[-1.5,0.4]$} & $0.326 \pm 0.231$ & $0.786 \pm 0.237$ & $0.112 \pm 0.061$ & $0.019 \pm 0.008$ & - & - & 0.01 & 2.1 & 279 \\
\hline$J-K_{\mathrm{s}}$ & {$[1.6,3.6]$} & {$[-4.8,1.0]$} & $-0.227 \pm 0.024$ & $0.466 \pm 0.021$ & $-0.023 \pm 0.005$ & $-0.016 \pm 0.002$ & $-0.005 \pm 0.001$ & - & 0.02 & 0.1 & 2180 \\
\hline$B_{\mathrm{T}}-V_{\mathrm{T}}$ & {$[1.6,2.4]$} & {$[-1.5,0.4]$} & $-0.247 \pm 0.023$ & $0.713 \pm 0.012$ & - & $0.175 \pm 0.007$ & - & - & 0.03 & 8.0 & 254 \\
\hline$g-r$ & {$[1.6,3.1]$} & {$[-2.4,0.4]$} & $-0.263 \pm 0.010$ & $0.521 \pm 0.005$ & - & $0.079 \pm 0.006$ & $0.015 \pm 0.004$ & - & 0.03 & 8.8 & 465 \\
\hline$g-i$ & {$[1.6,3.1]$} & {$[-1.4,0.4]$} & $0.280 \pm 0.084$ & $0.057 \pm 0.079$ & $0.163 \pm 0.018$ & $0.063 \pm 0.005$ & - & - & 0.03 & 13.5 & 282 \\
\hline$r-i$ & {$[1.6,3.1]$} & {$[-1.4,0.4]$} & $0.236 \pm 0.050$ & $-0.171 \pm 0.047$ & $0.095 \pm 0.011$ & - & - & - & 0.02 & 2.2 & 364 \\
\hline$G-W 1$ & {$[1.6,3.2]$} & {$[-2.4,0.5]$} & $0.099 \pm 0.043$ & $0.948 \pm 0.040$ & $0.019 \pm 0.009$ & $0.006 \pm 0.004$ & $0.007 \pm 0.003$ & - & 0.03 & 0.4 & 1666 \\
\hline$W 1-W 2$ & {$[1.6,3.2]$} & {$[-2.4,0.5]$} & $0.065 \pm 0.039$ & $-0.051 \pm 0.038$ & $-0.014 \pm 0.009$ & $0.049 \pm 0.015$ & $0.007 \pm 0.002$ & $-0.028 \pm 0.008$ & 0.02 & 0.1 & 1657 \\
\hline$W 2-W 3$ & {$[1.6,3.2]$} & {$[-2.4,0.5]$} & $-0.228 \pm 0.032$ & $0.240 \pm 0.029$ & $-0.038 \pm 0.006$ & - & - & - & 0.03 & 0.1 & 1671 \\
\hline$H-W 2$ & {$[1.6,3.2]$} & {$[-2.4,0.5]$} & $0.025 \pm 0.008$ & $0.032 \pm 0.004$ & - & $0.009 \pm 0.004$ & $0.016 \pm 0.003$ & - & 0.03 & 0.4 & 1137 \\
\hline
\end{tabular}

\subsection{Comparison with other studies}

In order to test the metallicity-dependent $T_{\text {eff }} C$ calibration derived in the current work, we took advantage of the already existing effective temperature relations provided by some studies. The closest literature relations to our $\hat{T}$ versus $G-K_{\mathrm{s}}$ calibration are $T_{\text {eff }}$ versus $V-K_{\mathrm{s}}$. We therefore selected a sample of APOGEE stars, with photometry information on the $G, V$, and $K_{\mathrm{s}}$ bands, which satisfy the quality criteria specified in Sect. 2. This gave us 179 stars for the test. Their effective temperatures were calculated using our $\hat{T}$ versus $G-K_{\text {s }}$ relation and through the $T_{\text {eff }}$ versus $V-K_{\mathrm{s}}$ relations from three different studies:

- Ramírez \& Meléndez (2005): They provided calibrations for main-sequence and giant stars based on temperatures that are derived with the infrared flux method (IRFM). These calibrations are valid within a range of temperatures and metallicities of $4000-7000 \mathrm{~K}$ and $-3.5 \leq[\mathrm{Fe} / \mathrm{H}] \leq 0.4$, respectively, and spectral types $\mathrm{F} 0$ to $\mathrm{K} 5$. The calibrations were carried out using a sample of more than 100 stars with known $U B V$, uvby, Vilnius, Geneva, RI (Cousins), DDO, HIPPARCOS-Tycho and 2MASS photometric bands.

- González Hernández \& Bonifacio (2009): they derived a new effective temperature scale for FGK stars, with the IRFM as well, using the 2MASS catalogue and theoretical fluxes computed from ATLAS models. Their $T_{\text {eff }}$-colour calibrations obtained with these temperatures are especially meant to be good for metal-poor stars. The calibrations were carried out via the Johnson-Cousins $B V(R I)$, 2MASS $J H K_{\text {s }}$ photometric bands, and Strömgren $b-y$ colour index

- Huang et al. (2015): they provided calibrations for dwarfs and giants that are based on a collection from the literature of about 200 nearby stars (including 54 giants) with direct interferometry effective temperature measurements. Their calibrations of giants are valid for an effective temperature range of 3100-5700 K and spectral types K5 to G5. The calibrations were carried out via the Johnson $U B V R I J H K$, Cousins $I_{\mathrm{C}} R_{\mathrm{C}}, 2 \mathrm{MASS} J H K_{\mathrm{s}}$, and SDSS gr photometric bands.

Figure 6 shows the residuals of the effective temperatures obtained with these various $T_{\text {eff }}$ versus $V-K_{\mathrm{s}}$ literature relations with respect to the $T_{\text {eff }}$ derived through our $\hat{T}$ versus $G-K_{\text {s }}$ fit. The differences stay mostly within $\pm 100 \mathrm{~K}$, but with a strong correlation with metallicity; these differences are mainly explained by the various sources of effective temperatures together with the different treatment of the interstellar extinction in each case. This correlation is stronger than that documented on the APOGEE DR13 release (see the corresponding discussion in Sect. 2.6). Applying the suggested correction still leads to a significant correlation with the residuals of the González Hernández \& Bonifacio (2009) temperatures, although smaller. For users interested in working in the González Hernández \& Bonifacio (2009) [GHB09] temperature scale, we found that, within the range of metallicity tested here, a simple linear relation allows the transformation $T_{\text {eff }(\mathrm{GHB} 09)}=$ $5040 \hat{T}+7-200[\mathrm{Fe} / \mathrm{H}]$.

\section{Red clump absolute magnitudes}

To calibrate the absolute magnitudes of the RC, we selected a different sample of stars. Indeed, in order to avoid contamination by the secondary red clump (see next Sect. 6), we made use only of stars within $1.93<G-K_{\mathrm{s}}<2.3$ and for which $M_{K_{\mathrm{S}}}$ is brighter than -0.5 (similar as in Eq. (2)). As in Sect. 2, we kept only low extinction stars (i.e. $\left.E(B-V)_{\max }<0.01\right)$ with $\sigma_{G}<0.01$ and high photometric quality on the $K$ band. The sample contains 2482 stars. For each band we then applied the same photometric constraints as for the $C C$ and $T_{\text {eff }} C$ calibrations, previously specified in Sect. 2.3.

Considering the strong contamination of the $\mathrm{RC}$ by the RGB bump and the variation of both the RC and RGB bump with colour, we did not estimate the RC absolute magnitude through a Gaussian fit to the magnitude distribution but through the mode of the distribution. The mode estimate is also less sensitive to the sample selection function. To model the colour dependency we looked for the maximum of $Q(\alpha, \beta)$, a kernel based distribution function of the residuals $M_{\lambda}-\left(\alpha\left(G-K_{\mathrm{s}}\right)+\beta\right)$, with $M_{\lambda}$ the absolute magnitude of each particular band,

$\max _{(\alpha, \beta)} Q(\alpha, \beta)=\sum_{i=1}^{N} \phi\left(\alpha+\beta\left(G-K_{\mathrm{s}}-2.1\right)-M_{\lambda}\right)$

where the constant 2.1 , the median of $G-K_{\mathrm{s}}$ of the sample, allows us to centre the fit on the RC.

The kernel $\phi$ we used corresponds to a Gaussian model of the parallax errors converted in magnitude space (we neglected 
L. Ruiz-Dern et al.: Gaia red clump photometric calibration
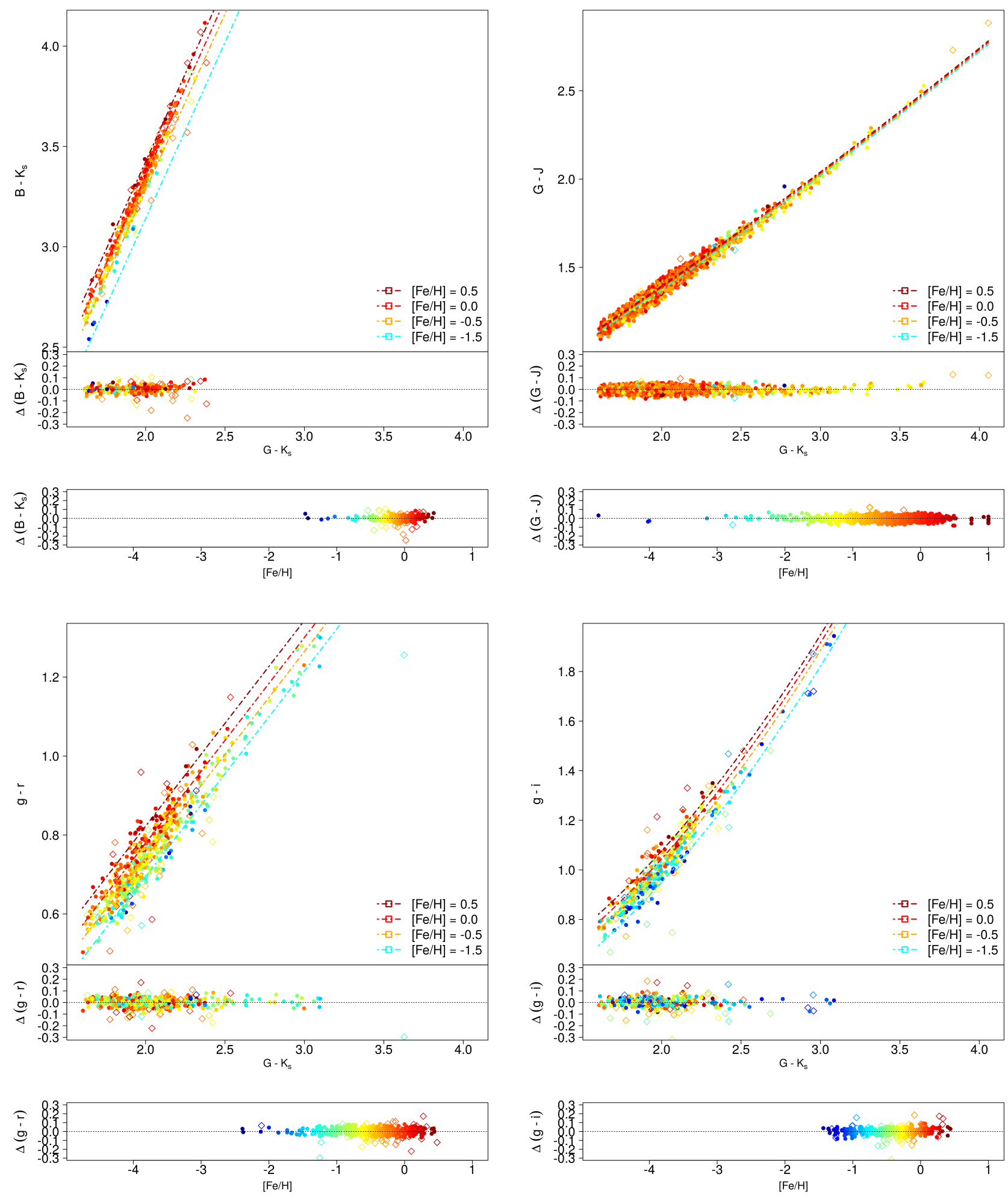

Fig. 4. Empirical colour vs. $G-K_{\mathrm{S}}$ calibrations for $B-K_{\mathrm{s}}, G-J, g-r$ and $g-i$ colour indices. The dash-dotted lines correspond to our calibration at fixed metallicities (see legend). The colour of the points varies as a function of the metallicity. Diamonds correspond to the outliers removed at $3 \sigma$ during the MCMC process. At the bottom of each calibration plot the residuals of the fit as a function of $G-K_{\mathrm{s}}$ and $[\mathrm{Fe} / \mathrm{H}]$ are shown. All plots are scaled to the same $G-K_{\mathrm{s}}$ colour and metallicity intervals.

here the photometric errors), i.e.

$\phi=P_{M}\left(M \mid M_{0}\right)=P_{\varpi}\left(\varpi(M) \mid \varpi_{0}\right) \frac{\partial \varpi}{\partial M}$

$\phi\left(\alpha+\beta\left(G-K_{\mathrm{s}}\right)-M_{\lambda}\right) \propto \mathcal{N}\left(\varpi_{\alpha, \beta, G}, \varpi, \sigma_{\varpi}\right) \varpi_{\alpha, \beta, m_{\lambda}}$ with $\varpi_{\alpha, \beta, m_{\lambda}}=10^{\left(\alpha+\beta\left(G-K_{\mathrm{S}}\right)-m_{\lambda}-5\right) / 5}$.
This method allows us to work directly with the parallaxes without selection in relative precision, avoiding the corresponding biases. 
Table 2. Coefficients and range of applicability of the $\hat{T}$ vs. $G-K_{\mathrm{S}}$ relation (top table) and the $\hat{T}$ vs. $G-K_{\mathrm{S}}$ relation (bottom table), $Y=$ $a_{0}+a_{1} X+a_{2} X^{2}+a_{3}[\mathrm{Fe} / \mathrm{H}]+a_{4}[\mathrm{Fe} / \mathrm{H}]^{2}+a_{5} X[\mathrm{Fe} / \mathrm{H}]$.

\begin{tabular}{|c|c|c|c|c|c|c|c|c|c|c|c|}
\hline$T_{\text {eff }}$ & $G-K_{\mathrm{s}}$ range & {$[\mathrm{Fe} / \mathrm{H}]$ range } & $a_{0}$ & $a_{1}$ & $a_{2}$ & $a_{3}$ & $a_{4}$ & $a_{5}$ & $\operatorname{RMS}_{\left[T_{\text {eff }}(K)\right]}$ & $\%_{\text {outliers }}$ & $N$ \\
\hline$\hat{T}$ & {$[1.6,3.7]$} & {$[-2.2,0.4]$} & $1.648 \pm 0.027$ & $-0.455 \pm 0.023$ & $0.054 \pm 0.005$ & $0.088 \pm 0.012$ & $0.001 \pm 0.002$ & $-0.026 \pm 0.006$ & 59 & 1.3 & 523 \\
\hline Colour & $T_{\text {eff }}$ range $(\mathrm{K})$ & {$[\mathrm{Fe} / \mathrm{H}]$ range } & $a_{0}$ & $a_{1}$ & $a_{2}$ & $a_{3}$ & $a_{4}$ & $a_{5}$ & $\operatorname{RMS}_{\left[G-K_{\mathrm{s}}\right]}$ & $\%_{\text {outliers }}$ & $N$ \\
\hline$G-K_{\mathrm{s}}$ & {$[3603.7,5207.7]$} & {$[-2.2,0.4]$} & $13.554 \pm 0.478$ & $-20.429 \pm 1.020$ & $8.719 \pm 0.545$ & $0.143 \pm 0.013$ & $-0.0002 \pm 0.009$ & - & 0.05 & 1.3 & 523 \\
\hline
\end{tabular}

Notes. We point out that $\hat{T}=T_{\text {eff }} / 5040$. The range of temperatures of the $\left[\hat{T}, G-K_{\mathrm{s}}\right]$ calibration (second table) is given in $T_{\text {eff }}($ not $\hat{T}$ ).
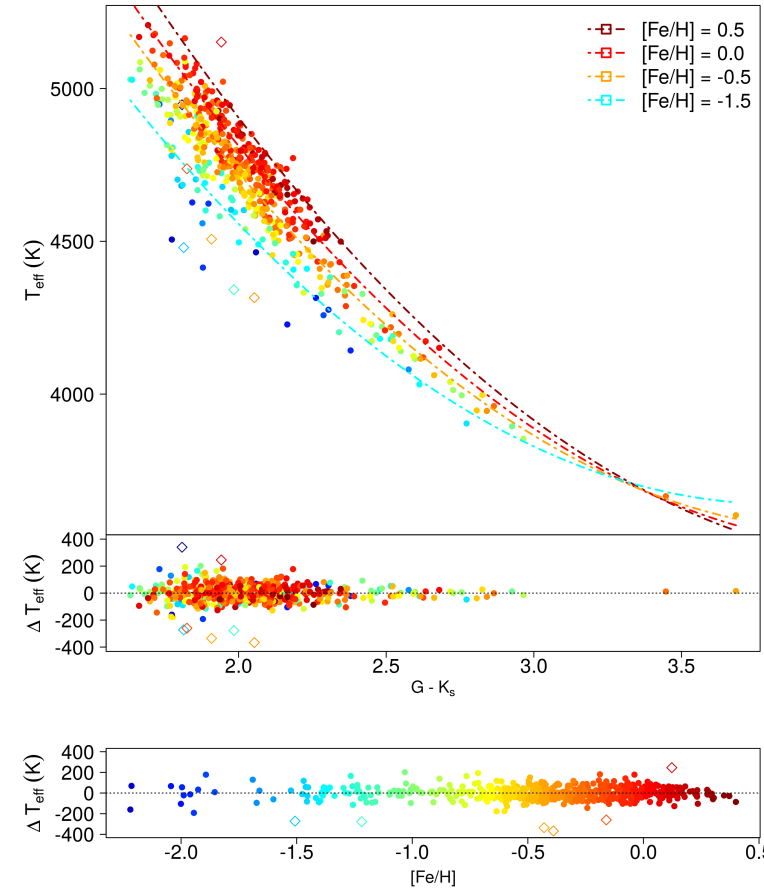

Fig. 5. Empirical normalised effective temperature vs. $G-K_{\mathrm{S}}$ calibration. The dash-dotted lines correspond to our calibration at fixed metallicities (see legend). The colour of the points varies as a function of the metallicity. Diamonds correspond to the outliers removed at $3 \sigma$ during the MCMC process. The residuals of the fit as a function of $G-K_{\mathrm{S}}$ and $[\mathrm{Fe} / \mathrm{H}]$ are shown at the bottom.

In Table 3 we summarise the results obtained with this method for 15 photometric bands. The initial uncertainties obtained through the maximum optimisation algorithm appeared underestimated ( 0.004). Indeed we saw that by changing the sample selection slightly, the results changed by more than the quoted errors. We provide in Table 3 the uncertainties by bootstrap instead.

We checked the degree of significance of the colour term for each relationship through a $p$-value test at $99 \%$ confidence level. We find a marginal dependence on colour for $M_{K_{\mathrm{S}}}$ ( $p$-value of 0.004 ) and for $M_{H}$ ( $p$-value of 0.002), negligible for $M_{W 1}, M_{W 2}$, $M_{W 3}$ and $M_{W 4}$, and an important dependence for $M_{G}$ and the other magnitudes. For those magnitudes for which there is no significant dependence we provide the results computed with $\beta$ fixed to zero (indicated with "-" in the table). We also include in Table 3 the results obtained for $M_{K_{\mathrm{S}}}$ and $M_{H}$ without taking into account their marginal dependence on colour $\left(M_{K_{\mathrm{S}}}=-1.606 \pm\right.$ $0.009, M_{H}=-1.450 \pm 0.017$ ).

We checked the robustness of the mode estimate versus the selected sample. We found differences of 0.006 mag when selecting only stars with $\sigma_{\varpi} / \varpi<10 \%$ (1085 stars).

We determined similarly the mode of the RC $M_{K_{\mathrm{S}}}$ distribution according to the Padova isochrones, simulating an HR

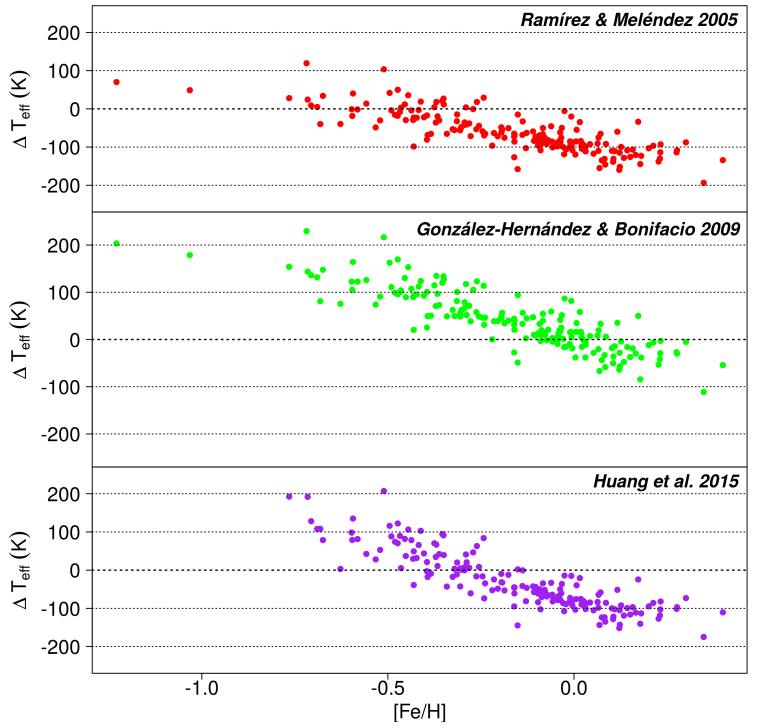

Fig. 6. Residuals of the effective temperatures obtained through the $T_{\text {eff }}$ vs. $V-K_{\mathrm{S}}$ calibrations of Ramírez \& Meléndez (2005) (top panel, red), González Hernández \& Bonifacio (2009) (middle panel, green), and Huang et al. (2015) (bottom panel, purple). These residuals are shown with respect to the values derived with the $\hat{T}$ vs. $G-K_{\mathrm{s}}$ fit of this work for a sample of 179 APOGEE stars with high photometric quality and low interstellar extinction.

diagram with a constant star formation rate (SFR), a Chabrier (2001) initial mass function (IMF), and a Gaussian metallicity distribution $(0,0.02)$. We obtained $M_{K_{\mathrm{S}}}=-1.660 \pm 0.003$, in agreement with Bovy et al. (2014). We checked that indeed the mode is robust to changes in the SFR, the IMF, and the agemetalliticy ratio (AMR) hypothesis.

A summary of various absolute magnitude calibrations in the literature can be found in Table 4, based on Table 1 of Girardi (2016) and complemented with more recent studies. In this table we indicate, for comparison purposes, our result from Table 3 assuming $G-K_{\mathrm{s}}$ colour equal to 2.1 when the external calibrations did not consider a colour effect while we found such a dependency.

We found general agreement with the $M_{K_{\mathrm{S}}}$ from previous works who mainly used HIPPARcos data. The $M_{K_{\mathrm{S}}}$ value of Alves (2000) is in the TMSS system (Bessell \& Brett 1988), while the others, including this work, mainly used 2MASS data. However the quality flags considered to select the data are not the same in each case. Our $M_{K_{\mathrm{S}}}$ value of the mean RC $K$-band absolute magnitude appears to be slightly lower than in Alves (2000), Grocholski \& Sarajedini (2002) and Laney et al. (2012), but higher than the values in van Helshoecht \& Groenewegen (2007), Groenewegen (2008) and Francis \& Anderson (2014). This value perfectly agrees with the last result of Hawkins et al. (2017) also using Gaia data but with a very different selection function and handling of the extinction. As in this work, 
Table 3. Coefficients of the absolute magnitude calibrations of the $\mathrm{RC}$, $M_{\lambda}=\alpha+\beta\left(G-K_{\mathrm{S}}-2.1\right)$.

\begin{tabular}{lccr}
\hline \hline$M_{\lambda}$ & $\alpha$ & $\beta$ & \multicolumn{1}{c}{$N$} \\
\hline$M_{B}$ & $1.931 \pm 0.009$ & $2.145 \pm 0.130$ & 1043 \\
$M_{V}$ & $0.855 \pm 0.009$ & $1.354 \pm 0.126$ & 1113 \\
$M_{B_{\mathrm{T}}}$ & $2.239 \pm 0.009$ & $2.397 \pm 0.135$ & 1190 \\
$M_{V_{\mathrm{T}}}$ & $0.975 \pm 0.009$ & $1.447 \pm 0.127$ & 1639 \\
$M_{g}$ & $1.331 \pm 0.056$ & $1.961 \pm 0.585$ & 407 \\
$M_{r}$ & $0.552 \pm 0.026$ & $1.194 \pm 0.289$ & 340 \\
$M_{i}$ & $0.262 \pm 0.032$ & $0.626 \pm 0.402$ & 243 \\
$M_{G}$ & $0.495 \pm 0.009$ & $1.121 \pm 0.128$ & 2482 \\
$M_{J}$ & $-0.945 \pm 0.010$ & $0.421 \pm 0.117$ & 2098 \\
$M_{H}$ & $-1.454 \pm 0.018$ & $0.234 \pm 0.224$ & 1315 \\
$M_{H}{ }^{*}$ & $-1.450 \pm 0.017$ & - & 1315 \\
$M_{K_{\mathrm{S}}}$ & $-1.605 \pm 0.009$ & $0.121 \pm 0.125$ & 2482 \\
$M_{K_{\mathrm{S}}}{ }^{*}$ & $-1.606 \pm 0.009$ & - & 2482 \\
$M_{W 1}$ & $-1.711 \pm 0.017$ & - & 962 \\
$M_{W 2}$ & $-1.585 \pm 0.016$ & - & 1031 \\
$M_{W 3}$ & $-1.638 \pm 0.011$ & - & 2026 \\
$M_{W 4}$ & $-1.704 \pm 0.012$ & - & 747 \\
\hline
\end{tabular}

Notes. ${ }^{(*)}$ Result without taking into account the marginal dependence on colour $(p$-value $<0.005)$

Groenewegen (2008) also found a weak dependency of $M_{K_{\mathrm{S}}}$ on colour.

For $M_{J}$, Laney et al. (2012) found a slightly larger result with respect to us. However, the source of photometric data is different from ours, and we have a much larger sample. Chen et al. (2017) used a much smaller sample and their value is even higher than that of Laney et al. (2012), but the former result is still consistent with this work. We find perfect agreement with Hawkins et al. (2017) who also used a sample of Gaia stars. The same authors also calibrated $M_{H}$, the results of which are in fair agreement with our value.

Chen et al. (2017) also calibrated the APASS-SLOAN gri absolute magnitudes using seismically determined RC stars from the Strömgren survey for Asteroseismology and Galactic Archaeology (SAGA). We find that the RC is less bright in all three magnitudes although within the errors bars.

As shown in Table 4, for $M_{W 1}$ our result agrees with both Chen et al. (2017) and Hawkins et al. (2017), and it is marginally brighter than that of Yaz Gökçe et al. (2013). For $M_{W 2}$ we also find good agreement with Chen et al. (2017), however the differences are larger with respect to Hawkins et al. (2017), as they have already pointed out in their article. We have indeed found important variations depending on the sample selection criteria. In particular, by considering only the high photometric quality flag (i.e. qph = A) and no cut in the observed magnitude, we obtained $M_{W 2}=-1.68 \pm 0.01$ with a strong correlation with colour. By removing the saturated stars (Cotten \& Song 2016), as described in Sect. 2.3, this dependence with colour becomes negligible, as it is for the other WISE bands, and the peak is much fainter. This may explain the too bright value found by Hawkins et al. (2017).

With $M_{W 3}$ all the works are consistent with the exception of Chen et al. (2017) who obtained a brighter value. And for $M_{W 4}$ our result is fainter than that found for the first time by Hawkins et al. (2017).

Finally, Hawkins et al. (2017) also provided the first calibration of $M_{G}$, based on a hierarchical probabilistic model. In this work we provide a different approach by directly using the mode of the distribution and with a larger sample of data. Their $G$ absolute magnitude is somewhat brighter. As mentioned above, with our method we also find a strong dependence on colour. This may explain the difference between both estimations, together with the fact that they corrected the reddening by deriving the extinction coefficients through the nominal Gaia $G$ band. The updated extinction coefficients will be found in Danielski et al. (in prep.).

Besides the parallax accuracy and various sources of photometric information, one of the main differences between these estimates is the handling of the interstellar extinction. Alves (2000), Stanek \& Garnavich (1998), Girardi et al. (1998), and Laney et al. (2012) assumed no reddening, while the other authors corrected their magnitudes using and combining different interstellar laws and/or maps. It is clear that our sample selection based on low extinction stars introduces as well a bias in all our calibrations, although minimally. Indeed, the reddening cut at $E(B-V)_{\max }<0.01$ corresponds to a maximum overestimation of the absolute magnitude of about $0.02 \mathrm{mag}$ in the $G$ band, while about 0.003 mag in the $K$ band (see Danielski et al., in prep.).

The discrepancies among the other estimates may also be justified by the different methods used: most of the authors considered a Gaussian fit, while here we used the mode of the distribution.

\section{The TGAS red clump HR diagram}

Figure 7 shows the TGAS HR diagram for red giant stars for absolute magnitudes in the $G$ and $K$ photometric bands. We used stars listed in Table A.1 (see Appendix A) with low extinction $\left(E(B-V)_{\max }<0.015\right), 10 \%$ parallax precision, $\sigma_{G}<0.01$, and 2MASS $J K$ high photometric quality. In both cases the $\mathrm{RC}$ is easily detected. However other features may also be observed. Indeed, on the bluest part of the RC we can see a small overdensity belonging to the secondary red clump (Girardi et al. 1998; Girardi 1999), a group of still metal-rich but younger (i.e. slightly more massive) stars that extend the RC to fainter magnitudes (up to 0.4 mag fainter). On the red side of the clump and below it, we find the red giant branch bump (RGB bump or RGBB), another overdensity of slightly more massive stars than the RC, which causes a peak (bump) in the luminosity function (see Christensen-Dalsgaard (2015) for a review on this CMD feature).

On the same diagrams we also overplotted the absolute magnitude calibrations obtained in previous Sect. 5 (Table 3).

In Fig. 8 we show the RC HR diagram again but with the Padova isochrones (Bressan et al. 2012, Parsec 2.7) at different metallicities (top panel) and at different ages (bottom panel) overplotted in different colours. We use the original $T_{\text {eff }}$ from the isochrones and applied our $G-K_{\mathrm{s}}$ versus $\hat{T}$ calibration (Table 2) to derive the colour $G-K_{\mathrm{s}}$.

We may see that while the position of the RC seems to nicely fit the isochrones, the RGB bump is slightly too bright in the isochrones. Following the process in Sect. 5, we found a difference of -0.07 mag between the Padova RC and the TGAS RC, and about 0.2 mag for the RGB bump.

\section{Conclusions}

Using the first Gaia Data Release parallaxes and photometry, the new 3D interstellar extinction map of Capitanio et al. (2017), the 2MASS catalogue and the last APOGEE release (DR13), a complete photometric calibration including colours (spread from 
Table 4. Comparison of the $M_{\lambda}$ of this work with other determinations in the literature.

\begin{tabular}{|c|c|c|c|c|}
\hline$M_{\lambda}$ & Reference & Calibration & Sample & $\begin{array}{l}\text { Extinction correction } \\
\end{array}$ \\
\hline \multirow[b]{2}{*}{$M_{G}$} & Hawkins et al. (2017) & $0.44 \pm 0.01$ & 972 TGAS (Gaia DR1) & $E(B-V)$ from 3D dustmap of Green et al. (2015) \\
\hline & This work & $0.495 \pm 0.009^{*}$ & 2482 TGAS (Gaia DR1) & $\begin{array}{l}\text { None: low extinction stars selection according } \\
\text { to Capitanio et al. (2017) 3D map }\end{array}$ \\
\hline \multirow{4}{*}{$M_{J}$} & Laney et al. (2012) & $-0.984 \pm 0.014$ & $\begin{array}{l}191 \text { Revised HIPPARCOS parallaxes with SAAO } J K \text { mag, } \\
\text { data corrected for Lutz-Kelker bias }\end{array}$ & None \\
\hline & Chen et al. (2017) & $-1.016 \pm 0.063$ & $\lesssim 171$ RC stars of the SAGA survey with 2MASS $J$ mag & SAGA $E(B-V)$ with Cardelli et al. (1989) law \\
\hline & Hawkins et al. (2017) & $-0.93 \pm 0.01$ & 972 TGAS ( Gaia DR1) with 2MASS $J$ mag & $E_{B-V}$ from 3D dustmap of Green et al. (2015) \\
\hline & This work & $-0.945 \pm 0.01^{*}$ & 2098 TGAS (Gaia DR1) with 2MASS $J$ mag & $\begin{array}{l}\text { None: low extinction stars selection according } \\
\text { to Capitanio et al. (2017) 3D map }\end{array}$ \\
\hline \multirow{4}{*}{$M_{H}$} & Laney et al. (2012) & $-1.490 \pm 0.015$ & $\begin{array}{l}191 \text { Revised HIPPARCOS parallaxes with SAAO } J K \text { mag, } \\
\text { data corrected for Lutz-Kelker bias }\end{array}$ & None \\
\hline & Chen et al. (2017) & $-1.528 \pm 0.055$ & $\lesssim 171$ RC stars of the SAGA survey with 2MASS $J$ mag & SAGA $E(B-V)$ with Cardelli et al. (1989) law \\
\hline & Hawkins et al. (2017) & $-1.46 \pm 0.01$ & 972 TGAS (Gaia DR1) with 2MASS $J$ mag & $E_{B-V}$ from 3D dustmap of Green et al. (2015) \\
\hline & This work & $-1.450 \pm 0.017$ & 1315 TGAS (Gaia DR1) with 2MASS $J$ mag & $\begin{array}{l}\text { None: low extinction stars selection according } \\
\text { to Capitanio et al. (2017) 3D map }\end{array}$ \\
\hline \multirow{9}{*}{$M_{K}$} & Alves (2000) & $-1.61 \pm 0.03$ & 238 HIPPARCOS RC giants with TMSS $K$ mag & None \\
\hline & Grocholski \& Sarajedini (2002) & $-1.61 \pm 0.04$ & 14 WYIN Open Clusters & Twarog et al. (1997) $E(B-V)$ with Cardelli et al. (1989) law \\
\hline & van Helshoecht \& Groenewegen (2007) & $-1.57 \pm 0.05$ & 24 2MASS Open Clusters & Twarog et al. (1997) $E(B-V)$ data \\
\hline & Groenewegen (2008) & $-1.54 \pm 0.04$ & Revised HIPPARCos parallaxes with 2MASS $K$ mag & Based on three 3D models \\
\hline & Laney et al. (2012) & $-1.613 \pm 0.015$ & $\begin{array}{l}191 \text { Revised HIPPARCOS parallaxes with SAAO } K \text { mag, } \\
\text { data corrected for Lutz-Kelker bias }\end{array}$ & None \\
\hline & Francis \& Anderson (2014) & $-1.53 \pm 0.01$ & Revised HIPPARCos parallaxes with 2MASS $K$ mag & $\begin{array}{l}\text { Outside } 100 \text { pc: Burstein \& Heiles (1978, 1982) map } \\
\text { and Bahcall \& Soneira (1980) formulae }\end{array}$ \\
\hline & Chen et al. (2017) & $-1.626 \pm 0.057$ & $\$ 171$ RC stars of the SAGA survey with 2MASS $K$ band & SAGA $E(B-V)$ with Cardelli et al. (1989) law \\
\hline & Hawkins et al. (2017) & $-1.61 \pm 0.01$ & 972 TGAS (Gaia DR1) with 2MASS $K$ mag & $E(B-V)$ from 3D dustmap of Green et al. (2015) \\
\hline & This work & $-1.606 \pm 0.009$ & 2482 TGAS (Gaia DR1) with 2MASS $K$ mag & $\begin{array}{l}\text { None: low extinction stars selection according } \\
\text { to Capitanio et al. (2017) 3D map }\end{array}$ \\
\hline \multirow[b]{2}{*}{$M_{g}$} & Chen et al. (2017) & $1.229 \pm 0.172$ & $\lesssim 171$ RC stars of the SAGA survey with APASS-SLOAN $g$ mag & SAGA $E(B-V)$ with Cardelli et al. (1989) law \\
\hline & This work & $1.331 \pm 0.056^{*}$ & 407 TGAS (Gaia DR1) with APASS-SLOAN $g$ mag & $\begin{array}{l}\text { None: low extinction stars selection according } \\
\text { to Capitanio et al. (2017) 3D map }\end{array}$ \\
\hline \multirow[b]{2}{*}{$M_{r}$} & Chen et al. (2017) & $0.420 \pm 0.110$ & $\lesssim 171$ RC stars of the SAGA survey with APASS-SLOAN $r$ mag & SAGA $E(B-V)$ with Cardelli et al. (1989) law \\
\hline & This work & $0.552 \pm 0.026^{*}$ & 340 TGAS ( Gaia DR1) with APASS-SLOAN $r$ mag & $\begin{array}{l}\text { None: low extinction stars selection according } \\
\text { to Capitanio et al. (2017) 3D map }\end{array}$ \\
\hline \multirow[b]{2}{*}{$M_{i}$} & Chen et al. (2017) & $0.157 \pm 0.094$ & $\$ 171$ RC stars of the SAGA survey with APASS-SLOAN $i$ mag & SAGA $E(B-V)$ with Cardelli et al. (1989) law \\
\hline & This work & $0.262 \pm 0.032^{*}$ & 243 TGAS (Gaia DR1) with APASS-SLOAN $i$ mag & $\begin{array}{l}\text { None: low extinction stars selection according } \\
\text { to Capitanio et al. (2017) 3D map }\end{array}$ \\
\hline \multirow{4}{*}{$M_{W 1}$} & Yaz Gökçe et al. (2013) & $-1.635 \pm 0.026$ & 3889 Revised HIPPARCOS RC parallaxes with WISE $W 1 \mathrm{mag}$ & $E(B-V)$ from 2D map of Schlegel et al. (1998) \\
\hline & Chen et al. (2017) & $-1.694 \pm 0.061$ & $\lesssim 171$ RC stars of the SAGA survey with WISE $W 1 \mathrm{mag}$ & SAGA $E(B-V)$ with Cardelli et al. (1989) law \\
\hline & Hawkins et al. (2017) & $-1.68 \pm 0.02$ & 936 TGAS ( Gaia DR1) with WISE $W 1 \mathrm{mag}$ & $E(B-V)$ from 3D dustmap of Green et al. (2015) \\
\hline & This work & $-1.711 \pm 0.017$ & 962 TGAS (Gaia DR1) with WISE $W 1$ mag & $\begin{array}{l}\text { None: low extinction stars selection according } \\
\text { to Capitanio et al. (2017) 3D map }\end{array}$ \\
\hline \multirow{3}{*}{$M_{W 2}$} & Chen et al. (2017) & $-1.595 \pm 0.064$ & $\lesssim 171$ RC stars of the SAGA survey with WISE $W 2 \mathrm{mag}$ & SAGA $E(B-V)$ with Cardelli et al. (1989) law \\
\hline & Hawkins et al. (2017) & $-1.69 \pm 0.02$ & 934 TGAS (Gaia DR1) with WISE $W 2 \mathrm{mag}$ & $E(B-V)$ from 3D dustmap of Green et al. (2015) \\
\hline & This work & $-1.585 \pm 0.016$ & 1031 TGAS (Gaia DR1) with WISE W2 mag & $\begin{array}{l}\text { None: low extinction stars selection according } \\
\text { to Capitanio et al. (2017) 3D map }\end{array}$ \\
\hline \multirow{4}{*}{$M_{W 3}$} & Yaz Gökçe et al. (2013) & $-1.606 \pm 0.024$ & 3889 Revised HIPPARCOS RC parallaxes with WISE $W 3$ mag & $E(B-V)$ from 2D map of Schlegel et al. (1998) \\
\hline & Chen et al. (2017) & $-1.752 \pm 0.068$ & $\lesssim 171$ RC stars of the SAGA survey with WISE $W 3 \mathrm{mag}$ & SAGA $E(B-V)$ with Cardelli et al. (1989) law \\
\hline & Hawkins et al. (2017) & $-1.67 \pm 0.02$ & 936 TGAS ( Gaia DR1) with WISE $W 3 \mathrm{mag}$ & $E(B-V)$ from 3D dustmap of Green et al. (2015) \\
\hline & This work & $-1.638 \pm 0.011$ & 2026 TGAS (Gaia DR1) with WISE W3 mag & $\begin{array}{l}\text { None: low extinction stars selection according } \\
\text { to Capitanio et al. (2017) 3D map }\end{array}$ \\
\hline \multirow[b]{2}{*}{$M_{W 4}$} & Hawkins et al. (2017) & $-1.76 \pm 0.01$ & 910 TGAS (Gaia DR1) with WISE W4 mag & $E(B-V)$ from 3D dustmap of Green et al. (2015) \\
\hline & This work & $-1.704 \pm 0.012$ & 747 TGAS (Gaia DR1) with WISE W4 mag & $\begin{array}{l}\text { None: low extinction stars selection according } \\
\text { to Capitanio et al. (2017) 3D map }\end{array}$ \\
\hline
\end{tabular}

Notes. ${ }^{(*)}$ Result from Table 3 assuming $G-K_{\mathrm{S}}$ colour equal to 2.1 .

visual to infrared wavelengths), absolute magnitudes, spectroscopic metallicities, and homogeneous effective temperatures is provided in this work for solar neighbourhood RC stars. We have made use of high photometric quality data from the Gaia, Johnson, 2MASS, HIPPARCOS, Tycho-2, APASS-SLOAN, and WISE photometric systems.

A robust MCMC method accounting for all variable uncertainties was developed to derive 20 accurate metallicity-dependent colour-colour relations and the $\hat{T}$ versus $G-K_{\mathrm{s}}$ and $G-K_{\mathrm{s}}$ versus $\hat{T}$ fits (with $\hat{T}$ the normalised effective temperature, $\left.\hat{T}=T_{\text {eff }} / 5040\right)$. We checked that the effective temperature calibration is compatible with those from Ramírez \& Meléndez (2005), González Hernández \& Bonifacio (2009) and Huang et al. (2015) within the metallicity and colour ranges of applicability.
We also derived the absolute magnitudes for the TGAS RC on 15 photometric bands (including $M_{G}$ and $M_{K_{\mathrm{S}}}$ ) through a kernel based magnitude distribution method and with the largest high quality dataset used so far for an absolute magnitude calibration of the RC. We obtained a small dependence on colour for $M_{K_{\mathrm{S}}}$ and $M_{H}$, which is not significant for $M_{W 1}, M_{W 2}, M_{W 3}$ and $M_{W 4}$, but important for $M_{G}$ and the other magnitudes.

All these photometric relationships will be improved in later Gaia releases and extended to other photometric bands, when larger RC samples will be available.

We presented a dereddened TGAS HR diagram for the $\mathrm{RC}$ region, in which we can already easily identify other features of red giant stars, such as the secondary RC and the RGB Bump. By using our calibrations we could compare the Padova isochrones with the TGAS HR diagram and found good 


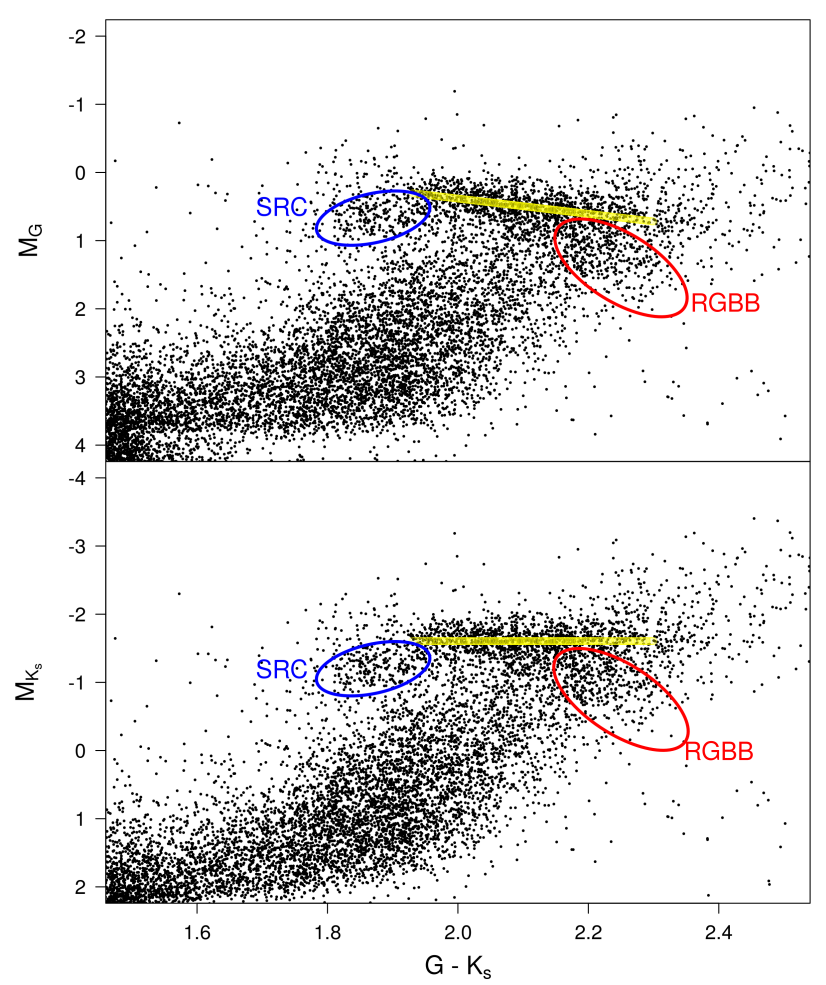

Fig. 7. TGAS HR diagram of the RC region for the $M_{G}$ (top) and the $M_{K_{\mathrm{S}}}\left(\right.$ bottom) absolute magnitudes, using stars with $E(B-V)_{\max }<$ $0.015,10 \%$ parallax precision, $\sigma_{G}<0.01$, and 2MASS $J K_{\mathrm{S}}$ high photometric quality. The location of the secondary red clump and the RGB bump features are easily observed on the diagram. We have highlighted them in blue and red, respectively. The yellow line shows the absolute magnitude calibration obtained in this work.

agreement with the RC location on the diagram, although the RGB bump appears too bright in the isochrones.

The photometric calibrations presented here are being used to derive $k_{G}$, the interstellar extinction coefficient in the $G$ band (Danielski et al., in prep.), and to provide photometric interstellar extinctions of large surveys such as APOGEE to be included in the next release of the new 3D extinction map of Capitanio et al. (2017).

In summary, this work used the high quality of the Gaia DR1 data to calibrate the Gaia RC. In turn, these calibrations can be used as the second rung of the cosmic distance ladder. Indeed, together with asteroseismic constraints, we can now derive the distance modulus of a large sample of RC stars. By choosing RC stars distant enough so that their estimated distance uncertainty is better than the Gaia parallax precision, these stars may be used to check the zero point of the Gaia parallaxes and their precision (Arenou et al. 2017). This is already being applied within the Gaia Data Release 2 verification process.

Acknowledgements. L.R.-D. acknowledges financial support from the Centre National d'Etudes Spatiales (CNES) fellowship programme. This work has made use of data from the European Space Agency (ESA) mission Gaia (https: //www.cosmos.esa.int/gaia), processed by the Gaia Data Processing and Analysis Consortium (DPAC, https://www.cosmos.esa.int/web/gaia/ $\mathrm{dpac}$ (consortium). Funding for the DPAC has been provided by national institutions, in particular the institutions participating in the Gaia Multilateral Agreement. This publication makes use of data products from the Two $\mathrm{Mi}$ cron All Sky Survey, which is a joint project of the University of Massachusetts and the Infrared Processing and Analysis Center/California Institute of Technology, funded by the National Aeronautics and Space Administration and the

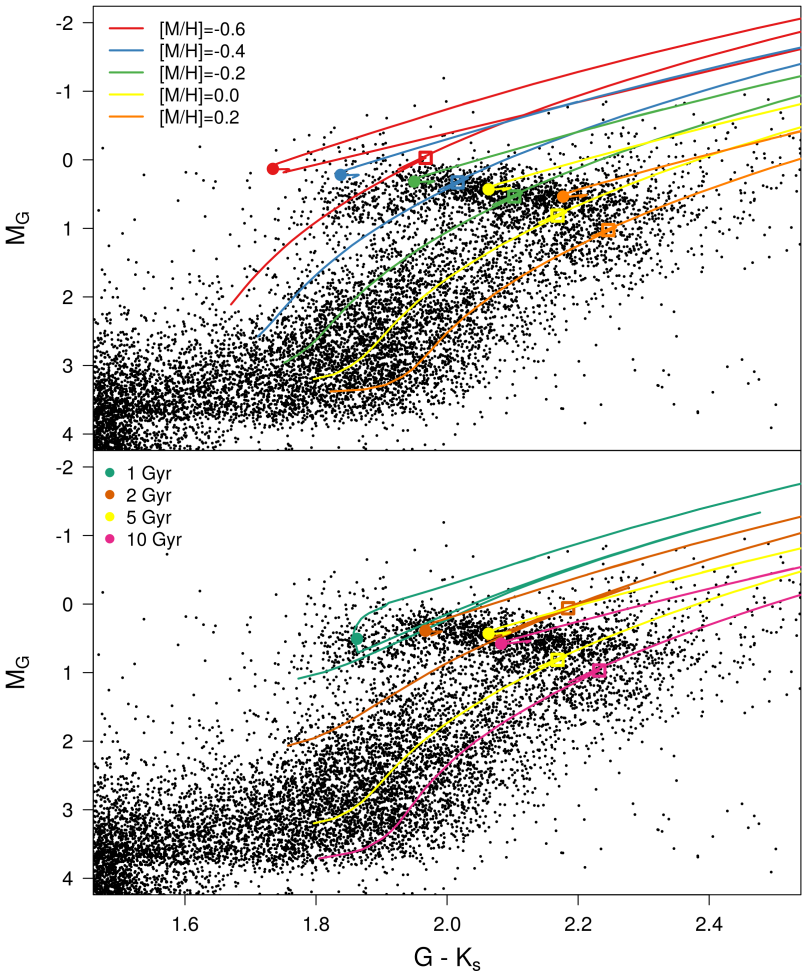

Fig. 8. Padova isochrones overlaid on the Gaia DR1 HRD. Top: isochrones for an age of $5 \mathrm{Gyr}$ and various metallicities are shown. Bottom: isochrones for a solar metallicity and various ages are shown. Circles correspond to the RC location and squares correspond to the RGB bump.

National Science Foundation. L.R.-D. acknowledges support from Agence Nationale de la Recherche through the STILISM project (ANR-12-BS05-0016-02). This research has also made use of VizieR databases operated at the Centre de Données astronomiques de Strasbourg (CDS) in France.

\section{References}

Adibekyan, V. Z., Sousa, S. G., Santos, N. C., et al. 2012, A\&A, 545, A32 Alves, D. R. 2000, ApJ, 539, 732

Alves, S., Benamati, L., Santos, N. C., et al. 2015, MNRAS, 448, 2749

Arenou, F., Luri, X., Babusiaux, C., et al. 2017, A\&A, 599, A50

Aringer, B., Girardi, L., Nowotny, W., Marigo, P., \& Bressan, A. 2016, MNRAS, 457, 3611

Bahcall, J. N., \& Soneira, R. M. 1980, ApJS, 44, 73

Bensby, T., Feltzing, S., \& Oey, M. S. 2014, A\&A, 562, A71

Bessell, M. S., \& Brett, J. M. 1988, PASP, 100, 1134

Bovy, J., Nidever, D. L., Rix, H.-W., et al. 2014, AJ, 790, 127

Bressan, A., Marigo, P., Girardi, L., et al. 2012, MNRAS, 427, 127

Bruntt, H., Basu, S., Smalley, B., et al. 2012, MNRAS, 423, 122

Burstein, D., \& Heiles, C. 1978, Astrophys. Lett., 19, 69

Burstein, D., \& Heiles, C. 1982, AJ, 87, 1165

Capitanio, L., Lallement, R., Vergely, J. L., Elyajouri, M., \& Monreal-Ibero, A. 2017, A\&A, 606, A65

Cardelli, J. A., Clayton, G. C., \& Mathis, J. S. 1989, AJ, 345, 245

Chabrier, G. 2001, ApJ, 554, 1274

Chen, Y. Q., Casagrande, L., Zhao, G., et al. 2017, ApJ, 840, 77

Christensen-Dalsgaard, J. 2015, MNRAS, 453, 666

Cotten, T. H., \& Song, I. 2016, ApJS, 225, 15

Cutri, R. M., Skrutskie, M. F., van Dyk, S., et al. 2003, VizieR Online Data Catalog: II/246

da Silva, R., Milone, A. d. C., \& Rocha-Pinto, H. J. 2015, A\&A, 580, A24

De Pascale, M., Worley, C. C., de Laverny, P., et al. 2014, A\&A, 570, A68

Evans, D. W., Riello, M., De Angeli, F., et al. 2017, A\&A, 600, A51

Fabricius, C., Høg, E., Makarov, V. V., et al. 2002, A\&A, 384, 180

Francis, C., \& Anderson, E. 2014, MNRAS, 441, 1105

Gaia Collaboration (Brown, A. G. A., et al.) 2016, A\&A, 595, A2

García Pérez, A. E., Allende Prieto, C., Holtzman, J. A., et al. 2016, AJ, 151, 144 
Girardi, L. 1999, in the evolution of the Milky Way: stars versus clusters (Kluwer Academic Publishers)

Girardi, L. 2016, ARA\&A, 54, 95

Girardi, L., Groenewegen, M. A. T., Weiss, A., \& Salaris, M. 1998, MNRAS 301,149

González Hernández, J. I., \& Bonifacio, P. 2009, A\&A, 497, 497

Green, G. M., Schlafly, E. F., Finkbeiner, D. P., et al. 2015, ApJ, 810, 25

Grocholski, A. J., \& Sarajedini, A. 2002, AJ, 123, 1603

Groenewegen, M. A. T. 2008, A\&A, 488, 935

Hawkins, K., Leistedt, B., Bovy, J., \& Hogg, D. W. 2017, MNRAS, 471, 722

Henden, A. A., Templeton, M., Terrell, D., et al. 2016, VizieR Online Data Catalog: II/336

Høg, E., Fabricius, C., Makarov, V. V., et al. 2000, A\&A, 355, L27

Holtzman, J. A., Shetrone, M., Johnson, J. A., et al. 2015, AJ, 150, 148

Huang, Y., Liu, X.-W., Yuan, H.-B., et al. 2015, MNRAS, 454, 2863

Jofré, E., Petrucci, R., Saffe, C., et al. 2015, A\&A, 574, A50

Jordi, C., Gebran, M., Carrasco, J. M., et al. 2010, A\&A, 523, A48

Kovtyukh, V. V., Soubiran, C., Bienaymé, O., Mishenina, T. V., \& Belik, S. I. 2006, MNRAS, 371, 879

Kunder, A., Kordopatis, G., Steinmetz, M., et al. 2017, AJ, 153, 75

Lallement, R., Vergely, J.-L., Valette, B., et al. 2014, A\&A, 561, A91

Laney, C. D., Joner, M. D., \& Pietrzynski, G. 2012, VizieR Online Data Catalog: J/MNRAS/419/1637

Lindegren, L., Lammers, U., Bastian, U., et al. 2016, A\&A, 595, A4

Luck, R. E. 2015, AJ, 150, 88

Luo, A.-L., Zhao, Y.-H., Zhao, G., et al. 2016, VizieR Online Data Catalog: V/149

Maldonado, J., \& Villaver, E. 2016, A\&A, 588, A98

Marrese, P. M., Marinoni, S., Fabrizio, M., \& Giuffrida, G. 2017, A\&A, 607, A105

Martell, S. L., Sharma, S., Buder, S., et al. 2017, MNRAS, 465, 3203

Morel, T., Miglio, A., Lagarde, N., et al. 2014, A\&A, 564, A119

Mortier, A., Santos, N. C., Sousa, S. G., et al. 2013, A\&A, 558, A106

Paczynski, B., \& Stanek, K. Z. 1998, ApJ, 494, L219

Perryman, M., Agency, E. S., \& Consortium, F. 1997, The HIPPARCos and Tycho Catalogues: The HIPPARCos catalogue, ESA SP (ESA Publications Division) Plummer, M. 2008, Biostatistics, 9, 523

Pourbaix, D., Tokovinin, A. A., Batten, A. H., et al. 2004, A\&A, 424, 727

Pourbaix, D., Tokovinin, A. A., Batten, A. H., et al. 2009, VizieR Online Data Catalog: B/sb9

Puzeras, E., Tautvaisiene, G., Cohen, J. G., et al. 2010, MNRAS, 408, 1225

Ramírez, I., \& Meléndez, J. 2005, ApJ, 626, 446

Ramirez, I., Melendez, J., \& Asplund, M. 2013, VizieR Online Data Catalog: J/A+A/561/A7
Ramírez, I., Bajkova, A. T., Bobylev, V. V., et al. 2014a, ApJ, 787, 154 Ramírez, I., Meléndez, J., Bean, J., et al. 2014b, A\&A, 572, A48

Saguner, T., Munari, U., Fiorucci, M., \& Vallenari, A. 2011, A\&A, 527, A40

Schlafly, E. F., \& Finkbeiner, D. P. 2011, ApJ, 737, 103

Schlegel, D. J., Finkbeiner, D. P., \& Davis, M. 1998, ApJ, 500, 525

SDSS Collaboration 2017, ApJS, 233, 25

Soubiran, C., Le Campion, J.-F., Brouillet, N., \& Chemin, L. 2016, A\&A, 591, A118

Stanek, K. Z., \& Garnavich, P. M. 1998, ApJ, 503, L13

Takeda, Y., Sato, B., \& Murata, D. 2008, PASJ, 60, 781

Thygesen, A. O., Frandsen, S., Bruntt, H., et al. 2012, A\&A, 543, A160

Twarog, B. A., Ashman, K. M., \& Anthony-Twarog, B. J. 1997, AJ, 114, 2556

Udalski, A. 2000, ApJ, 531, L25

Valentini, M., \& Munari, U. 2010, A\&A, 522, A79

van Helshoecht, V., \& Groenewegen, M. A. T. 2007, A\&A, 463, 559

van Leeuwen, F., Evans, D. W., De Angeli, F., et al. 2017, A\&A, 599, A32

Wright, E. L., Eisenhardt, P. R. M., Mainzer, A. K., et al. 2010, AJ, 140, 1868

Yaz Gökçe, E., Bilir, S., Öztürkmen, N. D., et al. 2013, New Astron., 25, 19

Zieliński, P., Niedzielski, A., Wolszczan, A., Adamów, M., \& Nowak, G. 2012, A\&A, 547, A91

\section{Appendix A: Low extinction TGAS HR Catalogue at CDS}

Table A.1 contains a few rows of the low extinction TGAS HRD compilation used in this work. The full table is available in VizieR.

The catalogue includes 142996 stars with

- Gaia DR1 and 2MASS identifiers.

- Gaia DR1 parallaxes with precision better than $10 \%$.

- Gaia DR1 $G$ magnitude with uncertainties lower than $0.01 \mathrm{mag}$.

- 2MASS $J$ and $K_{\mathrm{s}}$ photometric bands with high quality (i.e. flag q2M = "A.A") and uncertainties lower than $0.03 \mathrm{mag}$

- Reddening $E(B-V)_{\max }<0.015$ according to the Capitanio et al. (2017) 3D interstellar extinction map, and the Schlegel et al. (1998) 2D map for stars for which the distance goes beyond the 3D map borders.

Table A.1. First rows of the low extinction and high photometric and astrometric quality TGAS HRD catalogue.

\begin{tabular}{|c|c|c|c|c|c|c|c|c|c|c|}
\hline GDR1 id & 2MASS id & $\varpi$ & $\sigma_{\varpi}$ & $G$ & $\sigma_{G}$ & $J_{s}$ & $\sigma_{J_{s}}$ & $K_{s}$ & $\sigma_{K_{s}}$ & $E(B-V)_{\max }$ \\
\hline 7627862074752 & $03000819+0014074$ & 6.353 & 0.308 & 7.991 & 0.001 & 6.606 & 0.023 & 6.019 & 0.020 & 0.011 \\
\hline 16527034310784 & $03003397+0021355$ & 8.663 & 0.256 & 9.972 & 0.001 & 8.993 & 0.018 & 8.651 & 0.025 & 0.004 \\
\hline 26834955821312 & $03000244+0021039$ & 6.202 & 0.247 & 9.971 & 0.001 & 9.189 & 0.023 & 8.860 & 0.025 & 0.012 \\
\hline 44358422235136 & $03020031+0029521$ & 9.958 & 0.548 & 9.317 & 0.004 & 8.332 & 0.023 & 7.990 & 0.024 & 0.003 \\
\hline 115723598973952 & $03002534+0048455$ & 10.550 & 0.232 & 10.788 & 0.000 & 9.502 & 0.022 & 8.921 & 0.020 & 0.003 \\
\hline 122732985598464 & $03004702+0059362$ & 6.582 & 0.303 & 8.774 & 0.001 & 8.071 & 0.026 & 7.833 & 0.020 & 0.010 \\
\hline 308619170261760 & $02572363+0058185$ & 7.446 & 0.279 & 10.465 & 0.001 & 9.437 & 0.026 & 8.969 & 0.023 & 0.006 \\
\hline 310337157179392 & $02572548+0059538$ & 7.381 & 0.247 & 10.592 & 0.001 & 9.513 & 0.023 & 9.102 & 0.021 & 0.007 \\
\hline 320713798164992 & $02585888+0104389$ & 7.481 & 0.284 & 9.993 & 0.002 & 9.094 & 0.026 & 8.728 & 0.020 & 0.007 \\
\hline 349369819955456 & $02580800+0122163$ & 6.770 & 0.261 & 9.469 & 0.001 & 8.573 & 0.026 & 8.255 & 0.027 & 0.009 \\
\hline$\ldots$ & $\ldots$ & $\ldots$ & $\ldots$ & $\ldots$ & $\ldots$ & $\ldots$ & $\ldots$ & $\ldots$ & $\ldots$ & $\ldots$ \\
\hline
\end{tabular}

